\title{
Partial deletion of GLRB and GRIA2 in a patient with intellectual disability
}

\author{
Karl Hackmann ${ }^{\star, 1}$, Sarah Matko ${ }^{1}$, Eva-Maria Gerlach ${ }^{1}$, Maja von der Hagen ${ }^{2}$, Barbara Klink ${ }^{1}$, Evelin Schrock ${ }^{1}$, \\ Andreas Rump ${ }^{1}$ and Nataliya Di Donato ${ }^{1}$
}

We report about the partial de novo loss of GLRB and GRIA2 in an individual with intellectual disability (ID). No additional mutations were found in either gene. GLRB itself does not seem to be a good candidate as it causes autosomal recessive hyperekplexia and no symptoms were found in the patient. Mutations of GRIA2 have not been described as cause of ID to date. Nonetheless, it is a very attractive candidate because it encodes a subunit of a glutamate receptor, which is highly expressed in postsynaptic structures and has an important role in signal transduction across synapses. Although we were able to isolate a fragment of a fusion transcript of both genes from the patient's blood, we were not able to isolate a transcript with an open reading frame throughout the entire length. The reading frame could be restored by differential splicing, which might take place in brain tissue but not in blood. We assume that either haploinsufficiency of GRIA2 or a GLRBIGRIA2 fusion gene leading to a protein with dominant-negative properties is causing the phenotype of the patient.

European Journal of Human Genetics (2013) 21, 112-114; doi:10.1038/ejhg.2012.97; published online 6 June 2012

Keywords: GLRB; GRIA2; intellectual disability; fusion gene; glutamate receptor

\section{INTRODUCTION}

Glutamate receptors can be found in the dendritic part of synapses and aberrations in genes of glutamate receptor subunits have been identified as cause of intellectual disability (ID), that is, GRIN2A and GRIN2B, ${ }^{1}$ GRIA3 $3^{2,3}$ and GRIK2. ${ }^{4}$ GRIA2 encodes the GLUR2 subunit of AMPA ( $\alpha$-amino-3-hydroxy-5-methyl-4-isoxazolepropionic acid) receptors and is mainly expressed in brain. ${ }^{5}$ GLUR2 controls important biophysical properties of AMPA receptors and is involved in learning, memory and synaptic plasticity. ${ }^{6,7}$

\section{MATERIALS AND METHODS}

See Supplement.

\section{RESULTS}

Clinical presentation of the patient

The 3-year-old boy presented with severe speech delay, gait abnormalities (tip toeing) and abnormal behavior (hyperactivity, attention deficit and aggressive behavior). The family history is unremarkable. No structural malformations could be identified. The body measurements at birth and at the age of 3 years were normal. Some minor anomalies were seen but with the strong resemblance to both unaffected parents (detailed clinical information and facial view in Figure 1 Supplementary Information).

Molecular karyotyping

Array-CGH revealed, beside frequently observed variations, an unknown deletion on the long arm of chromosome 4 with a size of $102 \pm 9 \mathrm{~kb}($ arr 4q32.1 (158 267 339 × 2, 158272 983-158 $375402 \times 1$, $158378650 \times 2) \mathrm{dn})(\mathrm{NCBI} 36 / \mathrm{hg} 18)$.
Fluorescence in-situ hybridization (FISH)

FISH with fosmid G248P82551H7 (WI2-1059P14) (see Figure 1 for genomic position) confirmed the deletion in the patient. As the same probe gave regular signals on both chromosomes 4 of the parents (Figure 1), we conclude that the patient's deletion occurred de novo.

\section{Sequencing}

Sequencing of GRIA2 uncovered two homozygous SNPs in the patient's DNA: rs4302506 and rs4475186. Both SNPs have a minor allele frequency (MAF) of $T=0.362 / 456$ and are considered to be polymorphisms. Sequencing of GLRB uncovered three SNPs: rs12507409 was found in hemizygous state and results in a synonymous amino-acid exchange (MAF of $T=0.03 / 38$ ); rs41280499 (MAF of $T=0.452 / 568$ ) and rs3775724 (MAF of $T=0.375 / 472$ ) were found in heterozygous state. No SNPs were found that might reveal the parental origin of the chromosome carrying the deletion.

Expression of GLRB, GRIA2 and a fusion transcript in blood Amplification of commercial cDNA samples derived from normal brain (Clontech, Mountain View, CA, USA) with exon-based, GRIA2specific primers (Supplementary Table S1) resulted in several products of the GRIA2 transcript (data not shown). None of these amplifications were successful when tested on blood derived cDNAs obtained from control persons. GLRB could also easily be amplified from normal brain cDNA. In contrast to GRIA2, we found GLRB expression in blood. To see if a fusion transcript is being produced we used a forward primer in exon 4 of GLRB (and a reverse primer in exon 6 of GRIA2. A specific product could be amplified from the patient's blood cDNA, but not from the parents' blood cDNA

${ }^{1}$ Institut für Klinische Genetik, Medizinische Fakultät Carl Gustav Carus, Technische Universität Dresden, Dresden, Germany; ${ }^{2}$ Abteilung Neuropädiatrie, Klinik und Poliklinik für Kinder- und Jugendmedizin, Technische Universität Dresden, Dresden, Germany

*Correspondence: Dr K Hackmann, Institut für Klinische Genetik, Medizinische Fakultät Carl Gustav Carus, Technische Universität Dresden, Fetscherstr. 74 , 01307 Dresden, Germany. Tel: + 49351 4586861; Fax: +49 351 4586337; E-mail: karl.hackmann@tu-dresden.de

Received 5 September 2011; revised 18 April 2012; accepted 20 April 2012; published online 6 June 2012 


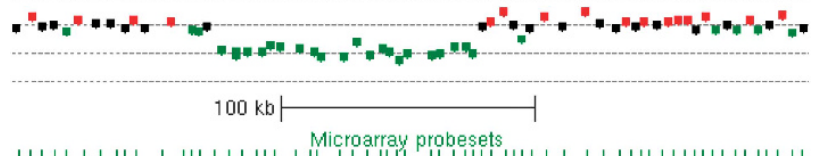

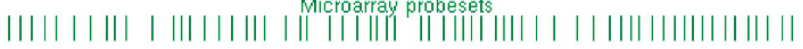
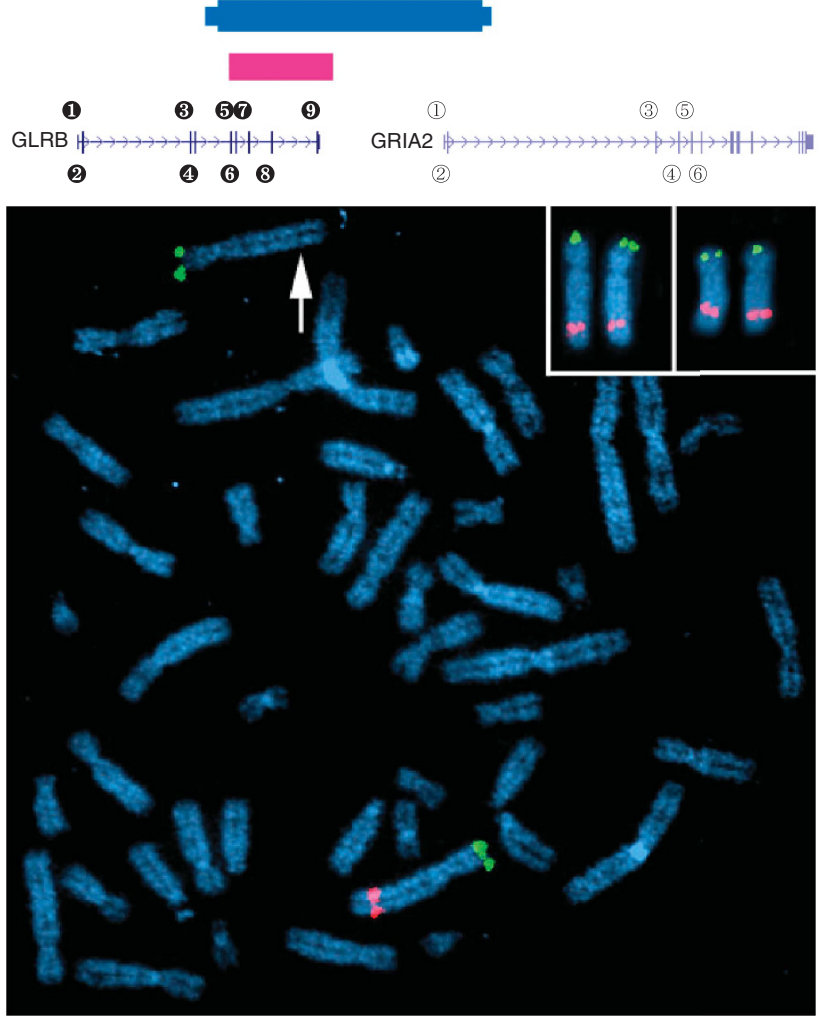

Figure 1 Partial deletion of GLRB and GRIA2, confirmed by array-CGH and FISH. Top: array-CGH data revealed an unknown deletion of $102 \pm 9 \mathrm{~kb}$, supported by 21 probes with significantly reduced signal intensity (green boxes on lower dashed line). Middle: UCSC-browser (hg19) diagram of the GLRB/GRIA2 region (chr4:157 970 647-158 289 501). The deletion (blue box, with thinner parts representing the breakpoint regions) removes exons 5-9 of GLRB (exon numbers in black circles) and exons 1-2 of GRIA2 (white circles). The pink box represents the genomic position of fosmid G248P82551H7, which was used in FISH. Bottom: absence of the fosmidspecific FISH-signal (pink) on one of the patient's chromosomes 4 (white arrow) validates the array-CGH data. As both chromosomes 4 (marked by a green chr4-specific subtelomeric probe) of each of the parents display the pink fosmid-signal (integrated boxes, upper right), the patient's deletion probably occurred de novo. The green vertical lines below the array data represent the genomic position of the corresponding probes on the Agilent SurePrint G3 Human CGH Microarray (Agilent, Santa Clara, CA, USA; $2 \times 400 \mathrm{~K}$, AMADID 021850).

(data not shown). Sequencing revealed a fusion transcript in which exon 4 of GLRB was spliced to exon 3 of GRIA2. The predicted result of this transcript fusion is a truncated GLRB protein (p.Met99fs) with an appendix of 20 amino acids of unique sequence (SAPSFREESMLFLDFMTRSL*).

\section{DISCUSSION}

Mutations in GLRB are associated with hyperekplexia. ${ }^{8,9}$ This disorder has been described as an autosomal recessive disorder and haploinsufficiency was excluded as possible cause. ${ }^{8,9}$ We assume that the partial deletion of GLRB itself does not have a major impact on the patient's ID, because no other mutations were found in this gene and no ID is described in patients with mutations in one or both allels of GLRB.

We hypothesize that GRIA2 is involved in the patient's ID. It was previously suggested from mouse models that GRIA2 might contribute to ID in Coffin-Lowry-syndrome. ${ }^{10}$ Tzschach et al ${ }^{11}$ reported on a patient with a balanced translocation $\mathrm{t}(2 ; 5)(\mathrm{p} 21 ; \mathrm{q} 12.1)$ and de novo loss of roughly $9 \mathrm{Mb}$ on chromosome 4 , including GLRB and GRIA2. The clinical presentations of this patient and our patient do not show specific overlapping abnormalities. However, it cannot be excluded that our patient might develop severe obesity in the second or third decade of life as the patient reported by Tzschach et al. ${ }^{11}$ They suggest haploinsufficiency of one or several genes as likely cause and GRIA2, GLRB, NPY1R and NPY5R as candidate genes that might cause the phenotype. No overlapping deletions were listed in the DECIPHER database by the end of October 2011. The ECARUCA database contains 26 cases with overlapping deletions. ${ }^{12}$ All but one are microscopically visible. One patient (ECARUCA ID 4689) with multiple malformations, short stature, microcephaly and ID is listed with a submicroscopic deletion with a size of $5.76 \mathrm{Mb}$ containing more than 30 genes.

The Database of Genomic Variants describes four copy number changes that are found in GRIA2 (variation no. 2551, 3520, 80534 and 92490), which are different from the one found in our patient. Each CNV was found in only one individual of the original 270 Hapmap samples or the Yoruban subgroup, respectively. ${ }^{13,14}$ None of these CNVs was reproduced faithfully even within the same work and the same samples. Instead two different duplications, one deletion and one inversion affecting GRIA2 were reported presumably for the same sample. As nothing is known about the individual the only conclusion that can be drawn is that CNVs in GRIA2 are rare.

Hamdan et $a l^{15}$ sequenced 197 genes that encode glutamate receptors in each of 95 sporadic cases of nonsymdromic ID. The supplementary information contains a homozygous mutation in GRIA2 (Asp781His) that was inherited from both parents and is predicted to be 'probably pathogenic' by Polyphen2 (http://genetics. bwh.harvard.edu/pph2/). There was no confirmation of or information on this result upon request. It remains open if the parents are affected or if this data has been validated.

Four genes that encode subunits of glutamate receptors have been described as a cause of nonsyndromic ID: GRIK2, GRIN2A, GRIN2B and GRIA3. ${ }^{1-4}$ Autosomal recessive (GRIK2), autosomal dominant (GRIN2A, GRIN2B) and X-linked (GRIA3) traits have been observed for these genes.

As we did not find a mutation in all exons and flanking intron areas of GRIA2 we have no evidence for an autosomal recessive mechanism in our patient. So haploinsufficiency of GRIA2 might be the underlying mechanism. The fusion transcript derived from GLRB and GRIA2 and detected only in the blood of our patient results in a frame shift mutation with a premature stop codon. An open reading frame might arise from various splice forms, for example, from exon 3 of GLRB spliced to exon 3 or 4 of GRIA2. We can neither prove nor exclude that this might happen in the patient's brain. A potential dominant-negative effect of the derivative fusion protein could explain the pathogenicity of this deletion. To our knowledge there is only one reported case of a fusion gene that causes ID. ${ }^{16}$

\section{CONFLICT OF INTEREST}

The authors declare no conflict of interest. 


\section{ACKNOWLEDGEMENTS}

The work was supported by the Bundesministerium für Bildung und Forschung (BMBF) network grant MR-NET 01GS08166.

1 Endele S, Rosenberger G, Geider K et al: Mutations in GRIN2A and GRIN2B encoding regulatory subunits of NMDA receptors cause variable neurodevelopmental phenotypes. Nat Genet 2010; 42: 1021-1026.

2 Wu Y, Arai AC, Rumbaugh G et al: Mutations in ionotropic AMPA receptor 3 alter channel properties and are associated with moderate cognitive impairment in humans. Proc Natl Acad Sci USA 2007; 104: 18163-18168.

3 Bonnet C, Leheup B, Beri M, Philippe C, Gregoire MJ, Jonveaux P: Aberrant GRIA3 transcripts with multi-exon duplications in a family with X-linked mental retardation. Am J Med Genet A 2009; 149A: 1280-1289.

4 Motazacker MM, Rost BR, Hucho T et al: A defect in the ionotropic glutamate receptor 6 gene (GRIK2) is associated with autosomal recessive mental retardation. Am J Hum Genet 2007; 81: 792-798.

5 Su Al, Wiltshire T, Batalov S et al: A gene atlas of the mouse and human proteinencoding transcriptomes. Proc Natl Acad Sci USA 2004; 101: 6062-6067.

6 Krugers HJ, Hoogenraad CC, Groc L: Stress hormones and AMPA receptor trafficking in synaptic plasticity and memory. Nat Rev Neurosci 2010; 11: 675-681.

7 Mead AN, Stephens DN: Involvement of AMPA receptor GluR2 subunits in stimulusreward learning: evidence from glutamate receptor gria2 knock-out mice. J Neurosci 2003; 23: 9500-9507.
8 Al-Owain M, Colak D, Al-Bakheet A et al: Novel mutation in GLRB in a large family with hereditary hyperekplexia. Clin Genet 2012; 81: 479-484.

9 Rees MI, Lewis TM, Kwok JB et al: Hyperekplexia associated with compound heterozygote mutations in the beta-subunit of the human inhibitory glycine receptor (GLRB). Hum Mol Genet 2002; 11: 853-860.

10 Mehmood T, Schneider A, Sibille J et al: Transcriptome profile reveals AMPA receptor dysfunction in the hippocampus of the Rsk2-knockout mice, an animal model of Coffin-Lowry syndrome. Hum Genet 2011; 129: 255-269.

11 Tzschach A, Menzel C, Erdogan F et al: Characterization of an interstitial 4q32 deletion in a patient with mental retardation and a complex chromosome rearrangement. Am J Med Genet A 2010; 152A: 1008-1012.

12 Feenstra I, Fang J, Koolen DA et al: European Cytogeneticists Association Register of Unbalanced Chromosome Aberrations (ECARUCA); an online database for rare chromosome abnormalities. Eur J Med Genet 2006; 49: 279-291.

13 Matsuzaki H, Wang PH, Hu J, Rava R, Fu GK: High resolution discovery and confirmation of copy number variants in 90 Yoruba Nigerians. Genome Biol 2009; 10: R125.

14 Redon R, Ishikawa S, Fitch KR et al: Global variation in copy number in the human genome. Nature 2006; 444: 444-454.

15 Hamdan FF, Gauthier J, Araki $Y$ et al: Excess of de novo deleterious mutations in genes associated with glutamatergic systems in nonsyndromic intellectual disability. Am J Hum Genet 2011; 88: 306-316.

16 Nothwang HG, Kim HG, Aoki J et al: Functional hemizygosity of PAFAH1B3 due to a PAFAH1B3-CLK2 fusion gene in a female with mental retardation, ataxia and atrophy of the brain. Hum Mol Genet 2001; 10: 797-806

Supplementary Information accompanies the paper on European Journal of Human Genetics website (http://www.nature.com/ejhg) 